\title{
Two New Phenolic Glycosides from Curculigo orchioides
}

\author{
Ai-Xue Zuo, ${ }^{\star}$ Yong Shen, ${ }^{\dagger}$ Zhi-Yong Jiang, ${ }^{\dagger}$ Xue-Mei Zhang, ${ }^{\ddagger}$ Jun Zhou, Jun Lü, ${ }^{\S}$ and Ji-Jun Chen ${ }^{\ddagger}, *$ \\ State Key Laboratory of Phytochemistry and Plant Resources in West China, Kunming Institute of Botany, \\ Chinese Academy of Sciences, Kunming, Yunnan 650204, China. *E-mail: aixuezuo@163.com \\ ${ }^{\dagger}$ Yunnan University of Traditional Chinese Medicine, 1076 Yu-Hua Road, Kunming 650500, China \\ ${ }^{*}$ Yunnan Agricultural University, Kunming 650201, China.*E-mail: chenjj@mail.kib.ac.cn \\ ${ }^{\S}$ Kunming Jingbiao Biosciences R\&D Co. Ltd., Kunming, Yunnan 650000, China \\ Received November 16, 2010, Accepted January 3, 2011
}

Two new phenolic glycosides were isolated from the rhizomes of Curculigo orchioides Gaertn.. Based on comprehensive spectroscopic analyses including IR, MS, 1D- and 2D NMR (COSY, HSQC, and HMBC), their structures were elucidated as 3-hydroxyl-5-methyphenol-1- $O$-[ $\beta$-D-glucopyranosyl- $(1 \rightarrow 3)$ - $\beta$-D-glucopyranoside (1) and 1',3'-dimethoxyl-4-hydroxyalangifolioside (2).

Key Words : Phenolic glycosides, Curculigo orchioides

\section{Introduction}

Curculigo orchioides Gaertn., belonged to the Amaryllidaceae family, was widely distributed in China, India, Malaya, Japan and Australia. ${ }^{1}$ The rhizomes of $C$. orchioides had been collected as a famous traditional Chinese medicine in the Chinese pharmacopeia. Previous phytochemical investigation on the rhizomes of $C$. orchioides revealed that it contained cycloartane triterpenes, ${ }^{2}$ phenolic glycosides, ${ }^{3}$ and chlorophenonic glycosides. ${ }^{4}$ Some chemicals from $C$. orchioides have exhibited stimulating immune response, ${ }^{5}$ antioxidative activities. ${ }^{6}$ During the last two years, our group had found some anti-depressant active phenolic compounds ${ }^{7}$ and several new phenolic glycoside dimers, ${ }^{8}$ phenolic glycosides $^{9,10}$ and cycloartane triterpenes ${ }^{11}$ from $C$. orchioides. As a further phytochemical investigation on this plant, two new phenolic glycosides, named 3-hydroxyl-5-methyphenol1- $O$-[ $\beta$-D-glucopyranosyl-(13)- $\beta$-D-glucopyranoside and 1',3-dimethoxyl-4-hydroxyalangifolioside were isolated from the $70 \%$ extract of the rhizomes of $C$. orchioides. This paper deals with the isolation, structure elucidation of two new phenolic glycosides based on spectroscopic techniques including MS, IR, 1D- and 2D NMR.

\section{Results and Discussion}

Compound 1 was obtained as colorless crystals $(\mathrm{MeOH})$ with an optical rotation of $[\alpha]_{\mathrm{D}}^{24.1}-42.42\left(c 0.32, \mathrm{C}_{5} \mathrm{H}_{5} \mathrm{~N}\right)$. Its molecular fomula was determined to be $\mathrm{C}_{19} \mathrm{H}_{28} \mathrm{O}_{12}$ on the basis of negative HR-ESI-MS at $m / z$ 447.1501 [M-H] ${ }^{-}$ (calcd for $\mathrm{C}_{19} \mathrm{H}_{27} \mathrm{O}_{12}, 447.1502$ ); The IR spectrum of compound 1 showed the absorption bands for hydroxyl group $\left(3364 \mathrm{~cm}^{-1}\right)$, aromatic ring $\left(1601,1507,1458 \mathrm{~cm}^{-1}\right)$, and glycosidic linkage $\left(1086 \mathrm{~cm}^{-1}\right)$ in the molecule. Hydrolysis of compound 1 with $2 \mathrm{M} \mathrm{H}_{2} \mathrm{SO}_{4}$ liberated glucose which was identified by comparing with the authentic sample on Paper Chromatography (PC) $\left[\mathrm{BuOH}-\mathrm{EtOAc}-\mathrm{H}_{2} \mathrm{O}\right.$
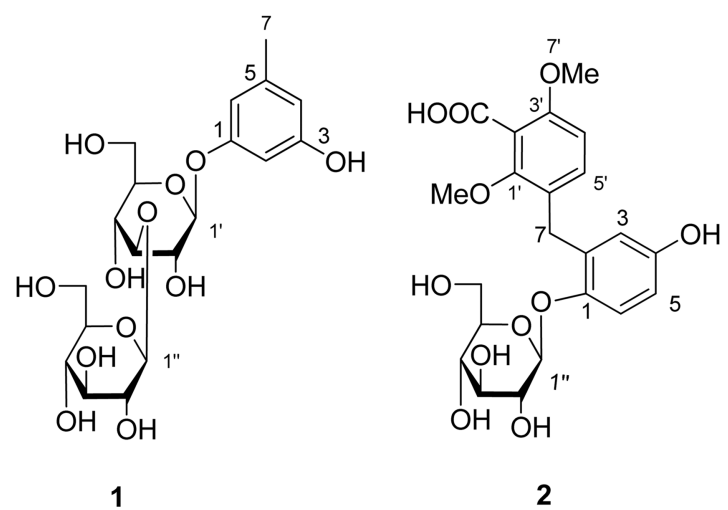

Figure 1. The structures of compounds 1-2.

4:1:5, upper layer; $\left.\mathrm{PhOH}-\mathrm{H}_{2} \mathrm{O}, 4: 1\right]$. In the ${ }^{1} \mathrm{H}-\mathrm{NMR}$ spectrum of compound $\mathbf{1}$, three aromatic proton signals corresponding to 1,3,5-trisubstituted aromatic ring at $\delta_{\mathrm{H}} 7.07(1 \mathrm{H}$, br. s), $6.80(1 \mathrm{H}$, br. s), $6.78(1 \mathrm{H}$, br. s), and one methyl resonance at $\delta_{\mathrm{H}} 2.19(3 \mathrm{H}, \mathrm{s})$ were observed, together with two anomeric proton signals at $\delta_{\mathrm{H}} 5.62(1 \mathrm{H}, \mathrm{d}, J=7.6 \mathrm{~Hz})$, $5.41(1 \mathrm{H}, \mathrm{d}, J=7.9 \mathrm{~Hz})$, suggesting the two glucose moieties in $\beta$-configuration. The ${ }^{13} \mathrm{C}$-NMR spectrum of compound 1 (Table 1) exhibited 19 carbon resonances, involving an aromatic ring: $\delta_{\mathrm{C}} 160.6(\mathrm{~s}), 102.6(\mathrm{~d}), 160.3(\mathrm{~s}), 109.2(\mathrm{~d})$, 140.0 (s), 111.7 (d); two glucopyranosyl moeities: $\delta_{\mathrm{C}} 102.2$ (d), $74.3(\mathrm{~d}), 88.7$ (d), 69.9 (d), 79.2 (d), 62.4 (t) and 106.4 (d), 76.2 (d), 78.8 (d), 72.1 (d), 78.8 (d), 63.0 (t), and one methyl $\delta_{\mathrm{C}} 22.2(\mathrm{q})$; Comparing the NMR data of compound 1 with those of orcinol glucoside ${ }^{12}$ revealed that compound 1 contained one more glucopyranose unit than orcinol glucoside; In order to determined the location of this additional glucopyranose moiety, an HMBC experiment was conducted. As shown in Figure 2, the HMBC correlation between H-1" $\left(\delta_{\mathrm{H}} 5.41,1 \mathrm{H}, \mathrm{d}, J=7.9 \mathrm{~Hz}\right)$ and $\mathrm{C}-3^{\prime}\left(\delta_{\mathrm{C}} 88.7, \mathrm{~d}\right)$ demonstrated the additional glucopyranose was linked at the C-3' of the inner glucopyranose; Therefore, compound 1 was 
Table 1. ${ }^{1} \mathrm{H}$ - and ${ }^{13} \mathrm{C}$ NMR data of compound 1 in $\mathrm{C}_{5} \mathrm{D}_{5} \mathrm{~N}(600 / 150 \mathrm{MHz} \delta$ in ppm, $J$ in $\mathrm{Hz})$

\begin{tabular}{|c|c|c|c|c|c|c|}
\hline No. & & & Glc-1' & & Glc-1" & \\
\hline & $\delta_{\mathrm{H}}$ & $\delta_{\mathrm{C}}$ & $\delta_{\mathrm{H}}$ & $\delta_{\mathrm{C}}$ & $\delta_{\mathrm{H}}$ & $\delta_{\mathrm{C}}$ \\
\hline 1 & & $160.6(\mathrm{~s})$ & $5.62(1 \mathrm{H}, \mathrm{d}, 7.6)$ & $102.2(d)$ & $5.41(1 \mathrm{H}, \mathrm{d}, 7.9)$ & $106.4(d)$ \\
\hline 2 & $7.07(1 \mathrm{H}$, br. s) & $102.6(d)$ & 4.34-4.35 (overlapped) & $74.3(\mathrm{~d})$ & $4.12-4.16(1 \mathrm{H}, \mathrm{m})$ & $76.2(d)$ \\
\hline 3 & & $160.3(\mathrm{~s})$ & 4.38-4.40 (overlapped) & $88.7(d)$ & $3.88-4.02(1 \mathrm{H}, \mathrm{m})$ & 78.8 (d) \\
\hline 4 & $6.80(1 \mathrm{H}$, br. s $)$ & $109.2(\mathrm{~d})$ & $4.26-4.28(1 \mathrm{H}, \mathrm{m})$ & $69.9(\mathrm{~d})$ & $4.23-4.24(1 \mathrm{H}, \mathrm{m})$ & 72.1 (d) \\
\hline 5 & & $140.0(\mathrm{~s})$ & $4.28-4.30(1 \mathrm{H}, \mathrm{m})$ & $79.2(d)$ & $4.28-4.30(1 \mathrm{H}, \mathrm{m})$ & 78.8 (d) \\
\hline 6 & $6.78(1 \mathrm{H}$, br. s) & $111.7(d)$ & $\begin{array}{l}4.38-4.40 \text { (overlapped) } \\
4.28-4.30(1 \mathrm{H}, \mathrm{m})\end{array}$ & $62.4(\mathrm{t})$ & $\begin{array}{l}4.59(1 \mathrm{H}, \mathrm{m}) \\
4.34-4.35 \text { (overlapped) }\end{array}$ & $63.0(\mathrm{t})$ \\
\hline 7 & $2.19(3 \mathrm{H}, \mathrm{s})$ & $22.2(q)$ & & & & \\
\hline
\end{tabular}
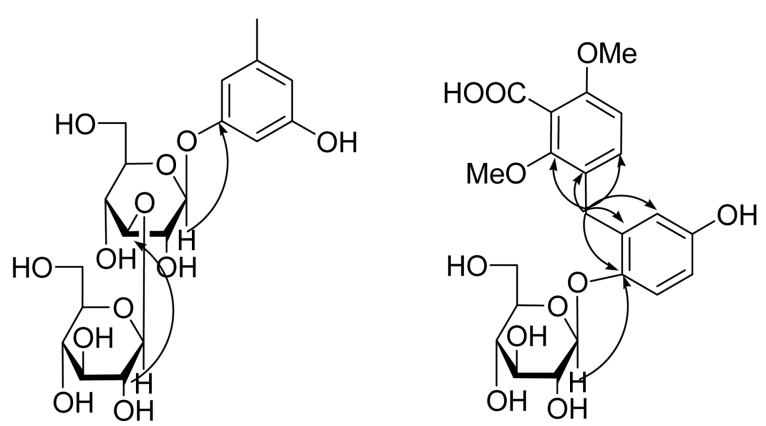

Figure 2. The key HMBC correlations of compounds 1-2.

elucidated as 3-hydroxyl-5-methyphenol-1- $O$-[ $\beta$-D-glucopyranosyl-( $1 \rightarrow 3)-\beta$-D-glucopyranoside.

Compound 2, a white amorphous powder, gave a molecular formula of $\mathrm{C}_{22} \mathrm{H}_{26} \mathrm{O}_{11}$ deduced by HR-EI-MS at $m / z 465.1400$ ([M-H] $]^{-}$; calcd. for $\left.\mathrm{C}_{22} \mathrm{H}_{25} \mathrm{O}_{11}, 465.1396\right)$ ); In the IR spectrum, the absorption bonds at $3407(\mathrm{OH}), 1703$ $(\mathrm{C}=\mathrm{O})$ and 1600, 1495, 1460 (aromatic ring) $\mathrm{cm}^{-1}$ were observed. Acidic hydrolysis of compound 2 afforded glucose identified by comparison with the authentic sample on PC [BuOH-EtOAc- $\mathrm{H}_{2} \mathrm{O}$ 4:1:5, upper layer; $\mathrm{PhOH}-\mathrm{H}_{2} \mathrm{O}$, 4:1]. The ${ }^{1} \mathrm{H}-\mathrm{NMR}$ demonstrated one typical 1,3,4-trisubstitued aromatic ring signals at $\delta_{\mathrm{H}} 6.43(1 \mathrm{H}, \mathrm{d}, J=2.8 \mathrm{~Hz})$,
$6.54(1 \mathrm{H}, \mathrm{dd}, J=8.8,2.8 \mathrm{~Hz}), 7.01(1 \mathrm{H}, \mathrm{d}, J=8.8 \mathrm{~Hz})$, one tetra-substitued aromatic ring protons resonances at $\delta_{\mathrm{H}} 6.65$ $(1 \mathrm{H}, \mathrm{d}, J=8.5 \mathrm{~Hz}), 6.97(1 \mathrm{H}, \mathrm{d}, J=8.5 \mathrm{~Hz})$, one methylene at $\delta_{\mathrm{H}} 3.95(2 \mathrm{H}, \mathrm{s})$, two methoxyls at $\delta_{\mathrm{H}} 3.80(3 \mathrm{H}, \mathrm{s}), 3.77$ $(3 \mathrm{H}, \mathrm{s})$, and a $\beta$-configuration anomeric proton at $\delta_{\mathrm{H}} 4.74$ $(1 \mathrm{H}, \mathrm{d}, J=7.3 \mathrm{~Hz})$; The ${ }^{13} \mathrm{C}-\mathrm{NMR}$ of compound 2 displayed 22 carbon signals including one methene at $\delta_{\mathrm{C}} 30.0(\mathrm{t})$, one carboxyl at $\delta_{\mathrm{C}} 175.0(\mathrm{~s})$, two methoxys at $\delta_{\mathrm{C}} 62.2(\mathrm{q}), 56.2$ (q), a set of glucopyranosyl moiety at $\delta_{\mathrm{C}} 104.1$ (d), 75.0 (d), 78.2 (d), $71.4(\mathrm{~d}), 78.0(\mathrm{~d}), 62.6(\mathrm{t})$, matched to those of $\beta$-methyl-glucopyranoside, ${ }^{13}$ as well as two aromatic rings (Table 2). Detailed analysis of the NMR data of compound $\mathbf{2}$ indicated that the compound $\mathbf{2}$ was structurally similar to 4-hydroxyalangifolioside. ${ }^{14}$ The main difference between them was that there were two additional methoxyl units in compound 2. The HMBC correlations from methoxyl signals at $\delta_{\mathrm{H}} 3.80(3 \mathrm{H}, \mathrm{s})$ and $3.77(3 \mathrm{H}, \mathrm{s})$ to $\delta_{\mathrm{C}} \mathrm{C}-1{ }^{\prime}(\mathrm{s}$, 155.6) and C-3' $(156.1, \mathrm{~s})$ constructed that the two additional methoxyls were located at C-1' and C-3', respectively. Based on the above evidences, compound $\mathbf{2}$ was characterized as 1',3'-dimethoxyl-4-hydroxyalangifolioside.

\section{Experimental}

General Experimental Procedures. Optical rotations

Table 2. ${ }^{1} \mathrm{H}$ - and ${ }^{13} \mathrm{C}$ NMR data of compound 2 in $\mathrm{CD}_{3} \mathrm{OD}(400 / 100 \mathrm{MHz} \delta$ in ppm, $J$ in $\mathrm{Hz})$

\begin{tabular}{|c|c|c|c|c|c|}
\hline No. & $\delta_{\mathrm{C}}$ & $\delta_{\mathrm{H}}$ & No. & $\delta_{\mathrm{C}}$ & $\delta_{\mathrm{H}}$ \\
\hline 1 & $150.2(\mathrm{~s})$ & & $1^{\prime}$ & $155.6(\mathrm{~s})$ & \\
\hline 2 & $133.8(\mathrm{~s})$ & & $2^{\prime}$ & $156.1(\mathrm{~s})$ & \\
\hline 3 & $117.7(d)$ & $6.43(1 \mathrm{H}, \mathrm{d}, 2.8)$ & $3^{\prime}$ & $127.2(\mathrm{~s})$ & \\
\hline 4 & $153.6(\mathrm{~s})$ & & $4^{\prime}$ & $107.8(d)$ & $6.65(1 \mathrm{H}, \mathrm{d}, 8.5)$ \\
\hline 5 & $114.2(d)$ & $6.54(1 \mathrm{H}, \mathrm{dd}, 8.8,2.8)$ & $5^{\prime}$ & $131.0(\mathrm{~d})$ & $6.97(1 \mathrm{H}, \mathrm{d}, 8.5)$ \\
\hline 6 & $118.4(d)$ & $7.01(1 \mathrm{H}, \mathrm{d}, 8.8)$ & $6^{\prime}$ & $126.3(\mathrm{~s})$ & \\
\hline 7 & $30.0(\mathrm{t})$ & $3.95(2 \mathrm{H}, \mathrm{s})$ & $7^{\prime}$ & $175.0(\mathrm{~s})$ & \\
\hline \multicolumn{6}{|l|}{ Glc } \\
\hline $1^{\prime \prime}$ & 104.1 (d) & $4.74(1 \mathrm{H}, \mathrm{d}, 7.3)$ & OMe-1' & $62.2(\mathrm{q})$ & $3.80(3 \mathrm{H}, \mathrm{s})$ \\
\hline $2^{\prime \prime}$ & $75.0(\mathrm{~d})$ & 3.43-3.47 (overlapped) & OMe-2' & $56.2(q)$ & $3.77(3 \mathrm{H}, \mathrm{s})$ \\
\hline $3^{\prime \prime}$ & $78.2(d)$ & 3.43-3.47 (overlapped) & & & \\
\hline $4^{\prime \prime}$ & $71.4(\mathrm{~d})$ & 3.37-3.38 (overlapped) & & & \\
\hline $5^{\prime \prime}$ & $78.0(\mathrm{~d})$ & 3.37-3.38 (overlapped) & & & \\
\hline \multirow[t]{2}{*}{$6^{\prime \prime}$} & $62.6(\mathrm{t})$ & $3.87(1 \mathrm{H}, \mathrm{dd}, 12.2,2.2)$ & & & \\
\hline & & $3.70(1 \mathrm{H}, \mathrm{m})$ & & & \\
\hline
\end{tabular}


were performed on a Horiba SEPA-300 polarimeter (Tokyo, Japan). IR spectra were recorded on a Bio-Rad FTS-135 spectrometer (Richmond, USA) with $\mathrm{KBr}$ pellets, $v$ in $\mathrm{cm}^{-1}$. UV spectra were measured on UV-210A spectrometer (Shimadzu, Japan); NMR spectra were conducted on Bruker AV-400 or DRX-600 spectrometers (Karlsruhe, Germany) with TMS as internal standard; chemical shift $(\delta)$ were expressed in ppm and coupling constants $(J)$ in Hz. FABMS was recorded on VG-Auto-spec-3000 mass spectrometer (Manchester, England); ESI and HR-ESI-MS were taken on a API Qstar-Pulsar-1 mass spectrometer (Applied Biosystems/MDS Sciex, Ontario, Canada). Column chromatography (CC) were performed on silica gel (200-300 mesh, Qingdao Meigao Chemical Co., Ltd., Qingdao, P.R. China), $\mathrm{Al}_{2} \mathrm{O}_{3}$ (Shanghai Wusi Chemical Reagents Company), $\mathrm{D}_{101}$ macroporous resins (Tianjin Pesticide Chemical Company), Sephadex LH-20 (Pharmacia Fine Chemical Co. Ltd. USA) and Lichroprep RP-18 (40-63 mm; Merck, Darmstadt, Germany); Fractions were monitored by TLC and visualization by spraying with $10 \% \mathrm{H}_{2} \mathrm{SO}_{4}$ in EtOH followed by heating.

Plant Material. The rhizomes of Curculigo orchioides Gaertn. were collected in Wenshan county, Yunnan Province, P. R. China, in November 2005, and authenticated by Prof. Dr. Li-Gong Lei, Kunming Institute of Botany, Chinese Academy of Sciences. A voucher specimen (NO. 20051106) had been deposited in the Group of Anti-virus and Natural Medicinal Chemistry, Kunming Institute of Botany, Chinese Academy of Sciences.

Extraction and Isolation. The air-dried and powdered rhizomes of C. orchioides ( $200 \mathrm{~kg}$ ) were extracted with $70 \%$ $\mathrm{EtOH}$ (each 1000L $2 \mathrm{~h}$ ) three times under reflux to yield an extract which was combined and concentrated to a small volume $(600 \mathrm{~L})$ and submitted to $\mathrm{CC}$ (macroporous resin D101, $200 \mathrm{~kg}$ ) with gradient elution of $\mathrm{H}_{2} \mathrm{O}, 10 \%$ EtOH$\mathrm{H}_{2} \mathrm{O}, 30 \%$ EtOH- $\mathrm{H}_{2} \mathrm{O}, 90 \%$ EtOH- $\mathrm{H}_{2} \mathrm{O}$ to afford four fractions: (Frs. A-D). The Fr. B (10\% EtOH- $\mathrm{H}_{2} \mathrm{O}$ eluted, 800 g) was subjected to $\mathrm{Al}_{2} \mathrm{O}_{3} \mathrm{CC}(8 \mathrm{~kg}, 14 \times 50 \mathrm{~cm})$ and subsequently eluted with EtOAc-EtOH- $\mathrm{H}_{2} \mathrm{O}$ (9:1:0.1), EtOAcEtOH- $\mathrm{H}_{2} \mathrm{O}(8: 2: 0.2)$ and EtOAc-EtOH- $\mathrm{H}_{2} \mathrm{O}(7: 3: 0.2)$ to afford sub-fractions B 1-3.

Fr. B $1(200 \mathrm{~g})$ was subjected to RP-18 CC $(1 \mathrm{~kg}, 6 \times 60$ $\mathrm{cm})$ eluted with $\mathrm{MeOH}-\mathrm{H}_{2} \mathrm{O}(2: 8)$ to afford fractions B1a-c, Fr. B 1a $(3.0 \mathrm{~g})$ was applied to a silica gel CC $(100 \mathrm{~g}$, $3.4 \times 27 \mathrm{~cm})$ eluted with $\mathrm{CHCl}_{3}-\mathrm{MeOH}-\mathrm{H}_{2} \mathrm{O}(8.5: 1.5: 0.15)$ to give four portions. The second portion $(1.2 \mathrm{~g})$ was purified on RP-18 CC $(120 \mathrm{~g}, 2.5 \times 33 \mathrm{~cm})$ eluted with $\mathrm{MeOH}-\mathrm{H}_{2} \mathrm{O}$ (3:97) to give a residue, which was purified by Sephadex LH-20 CC (53 g, $2.2 \times 62 \mathrm{~cm})$ eluted with $\mathrm{MeOH}$ to afford compound $2(21 \mathrm{mg})$. The Fr. B $1 \mathrm{c}(18.0 \mathrm{~g})$ was performed on silica gel CC $\left(250 \mathrm{~g}, 4 \times 50 \mathrm{~cm}, \mathrm{CHCl}_{3}\right.$ $\mathrm{MeOH}-\mathrm{H}_{2} \mathrm{O}$ 7:3:0.2) to give a residue $(1.2 \mathrm{~g})$ which was submitted to Sephadex LH-20 CC $(53 \mathrm{~g}, 2.2 \times 62 \mathrm{~cm}$, $\left.\mathrm{CHCl}_{3}-\mathrm{MeOH} 1: 1\right)$ and further purified by silica gel $\mathrm{CC}(15$ $\mathrm{g}, 1 \times 15 \mathrm{~cm})$ with the eluent of EtOAc-EtOH- $\mathrm{H}_{2} \mathrm{O}(8: 2: 0.2)$ to yield compound $\mathbf{1}(11 \mathrm{mg})$.

Compound (1): Colorless crystal $(\mathrm{MeOH}) ; \mathrm{C}_{19} \mathrm{H}_{28} \mathrm{O}_{12}$;
$[\alpha]_{\mathrm{D}}^{24.1}-42.42\left(c 0.32, \mathrm{C}_{5} \mathrm{H}_{5} \mathrm{~N}\right) ; \mathrm{UV}(\mathrm{MeOH}) \lambda_{\max }(\log \varepsilon)$ 273 (3.30) nm; IR (KBr) $v_{\max } 3364,2862,1601,1507,1458$, 1086, 1058, $574 \mathrm{~cm}^{-1}$; ${ }^{1} \mathrm{H}-$ and ${ }^{13} \mathrm{C}-\mathrm{NMR}$ see Table $1 ;(-)$ ESI-MS $m / z 447$ [M-H] $]^{-}$; (-) HR-ESI-MS $m / z 447.1501$ [M$\mathrm{H}]^{-}$(calcd for $\mathrm{C}_{19} \mathrm{H}_{27} \mathrm{O}_{12}, 447.1502$ ).

Compound (2): Amorphous powder; $\mathrm{C}_{22} \mathrm{H}_{26} \mathrm{O}_{11} ;[\alpha]_{D}^{21.4}$ -48.87 (c 0.12, MeOH); UV (MeOH) $\lambda_{\max }(\log \varepsilon) 284$ (3.70); IR (KBr) $v_{\max } 3407,2921,1703,1600,1495,1460$, 1396, 1213, 1081, 810, 609, $583 \mathrm{~cm}^{-1}$; ${ }^{1} \mathrm{H}-$ and ${ }^{13} \mathrm{C}-\mathrm{NMR}$ see Table 2; (-) FAB-MS $m / z 465[\mathrm{M}-\mathrm{H}]^{-}, 451[\mathrm{M}-\mathrm{Me}]^{-}$, 413 [M-Me-CO] $]^{-}, 399$ [M-Me-CO-CH$]^{-}, 381$ [M-Me-CO$\left.\mathrm{CH}_{2}-\mathrm{H}_{2} \mathrm{O}\right]^{-} ;(-)$HR-ESI-MS $m / z 465.1400\left([\mathrm{M}-\mathrm{H}]^{-}\right.$; calcd. for $\mathrm{C}_{22} \mathrm{H}_{25} \mathrm{O}_{11}$, calcd. for 465.1396).

Acid Hydrolysis. Each of compounds 1-2 (2 mg) was dissolved in $\mathrm{MeOH}(1.0 \mathrm{~mL})$ and $4 \mathrm{M} \mathrm{H}_{2} \mathrm{SO}_{4}(1.0 \mathrm{~mL})$ solution and hydrolyzed under reflux for $2 \mathrm{~h}$. The hydrolysate was allowed to cool, diluted with $2 \mathrm{~mL} \mathrm{H}_{2} \mathrm{O}$, and extracted with $2 \mathrm{~mL}$ EtOAc. The aq. layer was neutralized with aq. $\mathrm{Ba}(\mathrm{OH})_{2}$ and concentrated in vacuum to give a residue, in which glucose was identified by comparing with authentic sample on $\mathrm{PC}\left[\mathrm{BuOH}-\mathrm{EtOAc}-\mathrm{H}_{2} \mathrm{O}\right.$ 4:1:5, upper layer, $R_{f}=0.45 ; \mathrm{PhOH}-\mathrm{H}_{2} \mathrm{O}, 4: 1, R_{f}=0.40$ on PC respectively].

Acknowledgments. This work was financially supported by the project of Yunnan Science and Technology Plan (NO. 2008IF011), the major projects of new drugs development of China (No. 2009ZX09102-126), the 973 project of the Ministry of Sciences and Technology (No. 2009CB941300) and the Open Fund of the state key laboratory in KIB (NO. 0807E31211). The authors are grateful to the members of the staff of analytical group of State Key Laboratory of Phytochemistry and Plant Resources in West China, Kunming Institute of Botany, Chinese Academy of Sciences, for the measurements of all spectra.

\section{References}

1. Liu, G. W. Chinese Herbal Medicine; HuaXia Publishing House: Beijing, 2001; p 99.

2. Triguna, N. M.; Ram, S. S.; Deonath, M. T.; Shekhar, C. S. Phytochemistry 1990, 29, 929.

3. Kubo, M.; Namba, K.; Nagamoto, N.; Nagao, T.; Nakanishi, J.; Nishimura, H. Planta Med. 1983, 47, 52.

4. Xu, J. P.; Xu, R. S. Acta Pharm. Sin. 1992, 27, 353.

5. Lakshmi, V.; Pandey, K.; Puri, A.; Saxena, R. P.; Saxena, K. C. J. Ethnopharmacol. 2003, 89, 181.

6. Wu, Q.; Xu, F. D.; Hou, A. J.; Lei, G. Q.; Liu, Z. J.; Chen, J. K.; Zhou, T. S. Chem. Pharm. Bull. 2005, 53, 1065.

7. Chen, J. J.; Xu, L.; Zhou, J.; Lü, J.; Mao, R. R.; Tian, M.; Shen, Y.; Jiang, Z. Y. Chin. Pat. CN 101112367 2008, pp 1-21.

8. Zuo, A. X.; Shen, Y.; Jiang, Z. Y.; Zhang, X. M.; Zhou, J; Lü, J.; Chen, J. J. Helv. Chim. Acta 2010, 93, 504.

9. Zuo, A. X.; Shen, Y.; Jiang, Z. Y.; Zhang, X. M.; Zhou, J.; Lü, J.; Chen, J. J. J. Asian Nat. Prod. Res. 2010, 12, 43.

10. Zuo, A. X.; Shen, Y.; Jiang, Z. Y.; Zhang, X. M.; Zhou, J.; Lü, J.; Chen, J. J. Fitoterapia 2010, 81, 910.

11. Li, N.; Jia, A. Q.; Liu, Y. Q.; Zhou, J. Acta Bot. Yunnan 2003, 25, 241.

12. Li, N.; Zhao, Y. X.; Jia, A. Q.; Liu, Y. Q.; Zhou, J. Nat. Prod. Res. Dev. 2003, 15, 208.

13. Gorin, P. A. J.; Mazurek, M. Can. J. Chem. 1975, 53, 1212.

14. Tamaki, A.; Ide, T.; Otsuka, H. J. Nat. Prod. 2000, 63, 1417. 